\title{
Accurate broadband THz molecular spectroscopy
}

\author{
Krupnov A.F., Belov S.P., Tretyakov M.Yu., Golubiatnikov G.Yu., Parshin V.V., \\ Koshelev M.A., Serov E.A., Vilkov I.N., Makarov D.S., Bubnov G.M., \\ Leonov I.I., Chernova A.I., Andriyanov A.F., Shkaev A.P. \\ Institute of Applied Physics RAS, Nizhny Novgorod, Russia, koma@ipfran.ru
}

Teraherz (THz) molecular spectroscopy is a powerful instrument for solving both fundamental and applied problems. Molecular physics, remote sensing, chemical and isotopic analysis, medicine (breath analysis) are among the applied problems which can be solved using molecular spectroscopy methods.

Sensitivity, resolution and operating pressure range are the most crucial parameters of any spectrometer. High resolution of gas spectrometers allows observing the lines that uniquely as fingerprints characterize molecules, opening up vast possibilities, on the one hand, for the outlook at the molecular level, and, on the other hand, for qualitative and quantitative analysis of gas mixtures. High sensitivity of the spectrometer allows the significant expand of the range of the studied objects. The higher the sensitivity, the greater the number of lines which can be observed in the experiment. Finally, the wider the pressure range, the extensively the knowledge about intermolecular interaction which is required for adequate interpretation and modeling of the molecular spectra. However, use of only type one spectrometer cannot guarantee the data reliability. Use of several instruments of the different principal of operation allows obtaining more information on molecular spectra with higher accuracy and reliability.

In this report we present a complex of broadband $\mathrm{THz} / \mathrm{subTHz}$ spectrometers (see [1] and references therein) with complementary abilities developed in IAP RAS. The complex consists of three spectrometers with different methods of spectra recording covering the widest pressure range from a part of mTorr to a few atmospheres. All of them utilize backward wave oscillator (BWO) as a radiation source which frequency is stabilized by a phase lock loop (PLL) against a harmonic of a microwave frequency synthesizer and cover a frequency range from $30 \mathrm{GHz}$ to about $1 \mathrm{THz}$.

The first spectrometer is a conventional video spectrometer [2] operating in a pressure range from a part of mTorr to about 1 Torr. The principle of its operation consists in detection of a change of radiation power passed through the gas cell filled with the gas investigated. A spectrometer cell is a $2 \mathrm{~m}$ long and $113 \mathrm{~mm}$ diameter stainless-steel tube with polished internal cell surface. Optical vacuum windows are made of high-density polyethylene and are coneshaped to reduce the standing-wave amplitude between them. The spectrometer allows accurate measurement of line frequency, intensity, pressure broadening and shifting parameters. For precise measure- ment of line positions with subkHz uncertainty, method of nonlinear coherent spectroscopy (Lamb-dip method) was implemented in the spectrometer. A typical line recording obtained using video spectrometer is presented in Fig. 1.

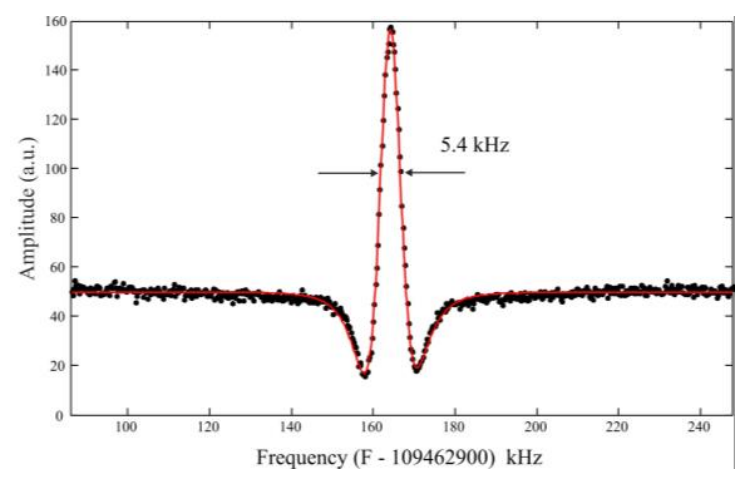

Fig. 1. Lamb-dip observed in OCS rotational transition $\mathrm{J}=9$ 8. Frequency detuning is shown from $109463.063 \mathrm{MHz}$. Collisional line width (HWHM) obtained from the fit to the Lorentz shape is $6.56(3) \mathrm{kHz}$. Doppler width is $81 \mathrm{kHz}$.

The second instrument of the spectroscopic complex is a spectrometer with radio-acoustic detector of absorption signal [3]. The output signal of the RAD spectrometer is proportional to the radiation power absorbed by the gas. It brings us to two main advantages of the RAD method: i) almost zero baseline (comparing to a video spectrometer); ii) the spectrometer sensitivity is increased as radiation power increases. The operating pressure range of the spectrometer is in interval from 10 mTorr to 10 Torr. The spectrometer is the best suited for the line shape analysis and measuring pressure broadening and shifting parameters of the lines and their speed-dependence in a wide temperature range. One more advantage of the RAD spectrometer is high enough sensitivity at rather compact size of the gas cell $(10 \mathrm{~cm}$ long and $2 \mathrm{~cm}$ in diameter). It allows easy shielding of the gas cell from the ambient magnetic fields that is important for studying the paramagnetic lines, e.g., for the lines of oxygen spectra. Typical recordings of oxygen lines obtained using RAD spectrometer are shown in Fig. 2.

At the present level of sensitivity of $\mathrm{THz}$ spectrometers most of spectroscopic studies are aimed at refinement of the spectroscopic information. Expanding the range of the studied objects requires significant improvement of the sensitivity. In $\mathrm{THz}$ range radio-acoustic detection is seemed to be the only method allowing reaching high sensitivity at reasonable recording time by increasing radiation power. It was demonstrated in our earlier study [4] that the use 
of the high power coherent $\mathrm{THz}$ radiation of the gyrotron allows increase of the RAD spectrometer sensitivity by at least three orders of magnitude. This opens up possibility of investigating, for example, the forbidden spectra of molecules.

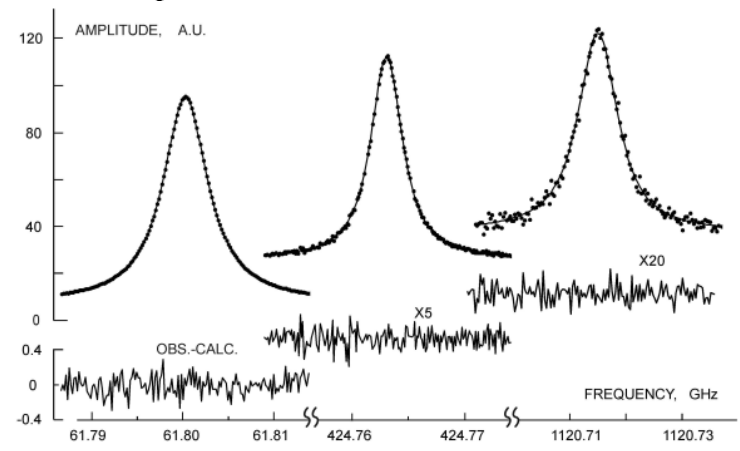

Fig. 2. Lines of the oxygen molecule obtained using RAD spectrometer (from left to right): a) fine-structure line 11+; b) rotational line $(\mathrm{N}, J)=(3,2)-(1,2)$; c) rotational line $(\mathrm{N}, \mathrm{J})=(7,6)-(5,6)$. The result of the line fitting to Voigt profile is shown by solid line. The residue of the fit is shown in the lower part of the figure.

In third, the resonator spectrometer [5,6] operating in a pressure range from 10 Torr up to a few atmospheres gas absorption is measured sequentially at each eigen-frequency as a change of the Q-factor of the Fabry-Perot resonator filled with the studied and non-absorbed gases, respectively. The PLL system provides fast phase-continuous frequency scan, resulted in significant progress in the spectrometer sensitivity (which now reaches $4 \cdot 10^{-9} \mathrm{~cm}^{-1}$ in terms of the absorption coefficient). A part of the atmospheric absorption spectra observed using resonator spectrometer is presented in Fig. 3. It is worth noting, that the line intensity measurements performed using the resonator spectrometer are supposed to be the most accurate comparing to other methods.

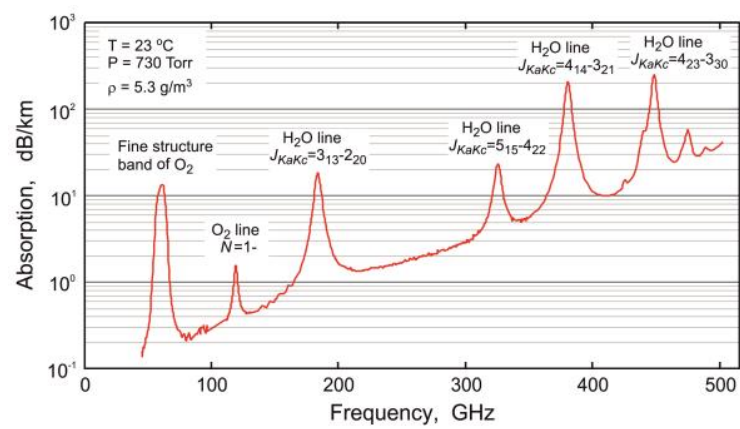

Fig. 3. Broadband experimental recording of atmospheric air spectrum obtained using resonator spectrometer.

Use of the complex of $\mathrm{THz}$ spectrometers allows determination of possible systematic errors. This is confirmed by a series of complementary experiments in which parameters of spectral lines measured by one spectrometer were revised by a spectrometer of another type. Coincidence of the values of the parameters measured by different spectrometers means high accuracy and reliability of the data obtained. As an example, results of the pressure broadening study for $118-\mathrm{GHz}$ oxygen line [7] (RAD and resonator) and
22-GHz water line [8] (RAD and video) are presented in Fig. 4.
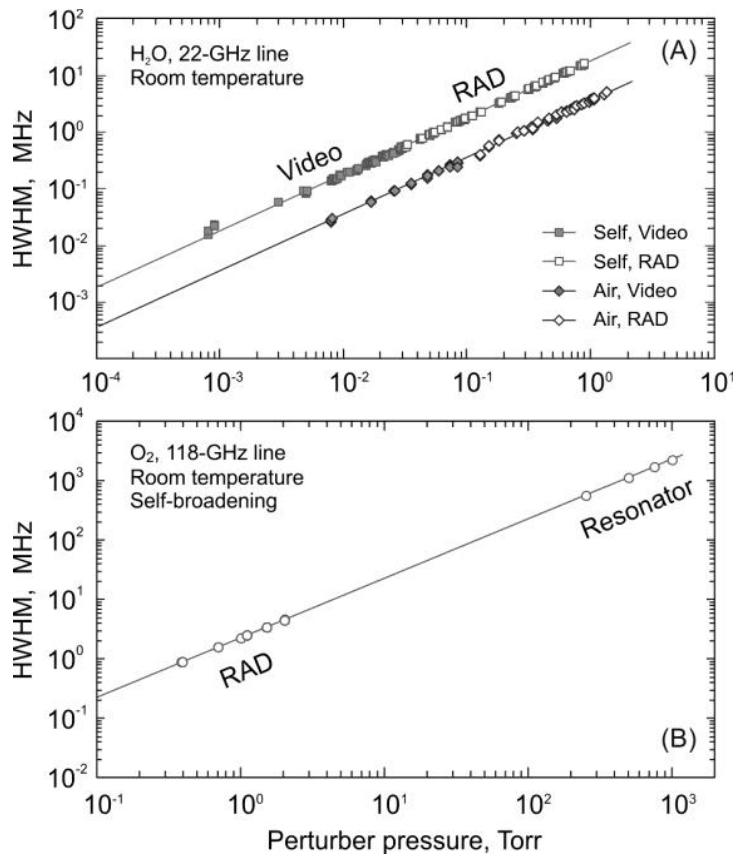

Fig. 4. Pressure dependence of the width of the (A) $22-\mathrm{GHz}$ water line measured using video and RAD spectrometers and (B) 118-GHz oxygen line measured using RAD and resonator spectrometers.

Improvement of the RAD spectrometer sensitivity is supported by RSF (project 17-19-01602). Studies of molecular spectra using complex of spectrometers are supported in parts by RFBR projects 18-05-00698 (atmospheric pressure recordings) and 18-02-00705 (line shape analysis).

\section{References}

1. Krupnov A.F., et al. Accurate broadband rotational BWO-based spectroscopy // J. Molec. Spectrosc. 2012. V. 280 P.110-118.

2. Golubiatnikov G.Yu., et al. Precision Sub-Doppler Millimeter and Submillimeter Lamb-Dip Spectrometer // Radiophysics and Quantum Electronics. 2014. V 56. N. 8-9. P. 599-609.

3. Tretyakov M.Yu., et al. Precise Measurements of Collision Parameters of Spectral Lines with a Spectrometer with Radioacoustic Detection of Absorption in the Millimeter and Submillimeter Ranges // Instrum. Exp. Tech. 2008. V. 51. P. 78-88.

4. Koshelev M.A., et al. Molecular Gas Spectroscopy Using Radioacoustic Detection and High-Power Coherent Subterahertz Radiation Sources // J. Molec. Spectrosc. 2017. V. 331 P. 9-16.

5. Parshin V.V., et al. Modern resonator spectroscopy at submillimeter wavelengths. // IEEE Sensors Journal. 2013 V.13. N. 1. P. 18-23.

6. Koshelev M.A., et al. New frontiers of modern resonator spectroscopy // submitted in IEEE Trans. THz Sci. Technol. 2018.

7. Koshelev M.A., et al. Accurate modeling of the diagnostic $118-\mathrm{GHz}$ oxygen line for remote sensing of the atmosphere // J. Quant. Spectrosc. Radiative Transfer 2017. V. 196 P. 78-86.

8. Koshelev M.A., et al. Line shape parameters of the 22-GHz water line for accurate modeling in atmospheric applications // J. Quant. Spectrosc. Radiative Transfer 2018. V. 205 P. 51-58. 